\title{
The Effect of 50000 IU Vitamin A with BCG Vaccine at Birth on Growth in the First Year of Life
}

\author{
Ane Bærent Fisker, ${ }^{1,2,3}$ Christine Stabell Benn, ${ }^{1,2}$ Birgitte Rode Diness, ${ }^{1}$ \\ Cesario Martins, ${ }^{1}$ Amabelia Rodrigues, ${ }^{1}$ Peter Aaby, ${ }^{1,2}$ and Bo Martin Bibby ${ }^{3}$ \\ ${ }^{1}$ Bandim Health Project, Indepth Network, Apartado 861, 1004 Bissau, Guinea-Bissau \\ ${ }^{2}$ Bandim Health Project, Statens Serum Institut, Ørestads Boulevard 5, 2300 Copenhagen S, Denmark \\ ${ }^{3}$ Department of Biostatistics, Institute of Public Health, University of Århus, Bartholins Alle 2, 8000 Århus C, Denmark
}

Correspondence should be addressed to Ane Bærent Fisker, a.fisker@bandim.org

Received 23 March 2011; Revised 2 July 2011; Accepted 6 July 2011

Academic Editor: Sabra Klein

Copyright (C) 2011 Ane Bærent Fisker et al. This is an open access article distributed under the Creative Commons Attribution License, which permits unrestricted use, distribution, and reproduction in any medium, provided the original work is properly cited.

\begin{abstract}
Vitamin A supplements may interact with diphtheria-tetanus-pertussis (DTP) vaccine causing increased female mortality. In a randomised trial of neonatal vitamin A supplementation (VAS), we examined growth during the first year of life in 808 children, pursuing the hypothesis that a negative interaction between VAS and DTP in girls would be reflected in growth. Length and weight were measured at 6 weekly visits and WHO-growth-reference z-scores derived. Neonatal VAS had no effect on anthropometric measures at 12 months, but may interact sex differentially with routine vaccines. While BCG was the most recent vaccine, neonatal VAS benefitted growth (difference in weight-for-length z-score (dWFL: 0.31(95\% CI: 0.03-0.59)). While DTP was the most recent vaccine, VAS tended to affect growth adversely in girls ( dWFL $=-0.21(-0.48-0.06)$ ). After measles vaccine $(\mathrm{MV})$ there was no overall effect of neonatal VAS. The VAS effect differed significantly between the BCG and DTP windows $(P=0.03)$, and the difference was borderline significant between the DTP and MV windows for girls $(P=0.09)$.
\end{abstract}

\section{Introduction}

Vitamin A deficiency (VAD) has been associated with impaired growth in observational studies [1,2], but randomised trials of vitamin A supplementation (VAS) have often failed to show an impact on growth [3] in spite of improved vitamin A status in deficient populations $[4,5]$. The only study examining the effect of neonatal VAS on growth reported a positive effect on height at 3 years of age in Indonesia [6]. Within a randomised placebo-controlled study of the effect on mortality of providing neonates with $50000 \mathrm{IU}$ of vitamin A with Bacillus Calmette-Guerin vaccine (BCG) [7] we examined the effect of neonatal VAS on growth during the first year of life in both sexes.

The present trial as well as a similar trial of neonatal VAS from Zimbabwe [8] did not find the expected beneficial effect of VAS on mortality observed in Indonesia [9] and India [10] and later in Bangladesh [11]. We have previously hypothesised that VAS and diphtheria-tetanus-pertussis (DTP) vaccines may interact negatively $[12,13]$, due to amplification of the negative nonspecific effects of DTP vaccines.

The nonspecific effects are the effects of vaccines that cannot be ascribed to the protection against the targeted disease(s). For example, observational studies $[14,15]$ and randomised trials $[16,17]$ of measles vaccine (MV) indicate reductions in overall mortality far bigger than the reduction due to prevention of measles infection. Similarly for BCG, reductions in mortality that cannot be ascribed to prevention of tuberculosis have been seen in randomised $[18,19]$ and observational studies [20]. The opposite effect is seen after DTP vaccinations; in areas with herd immunity to pertussis, DTP has been associated with increased female mortality reflected in increased mortality in DTP vaccinated compared to unvaccinated children [15] and increased female-male mortality rate ratio after DTP vaccination $[21,22]$. In the present cohort we found that girls had 2-fold higher mortality after reception of DTP vaccine if they had received VAS rather than placebo at birth [23]. In the present study, we 


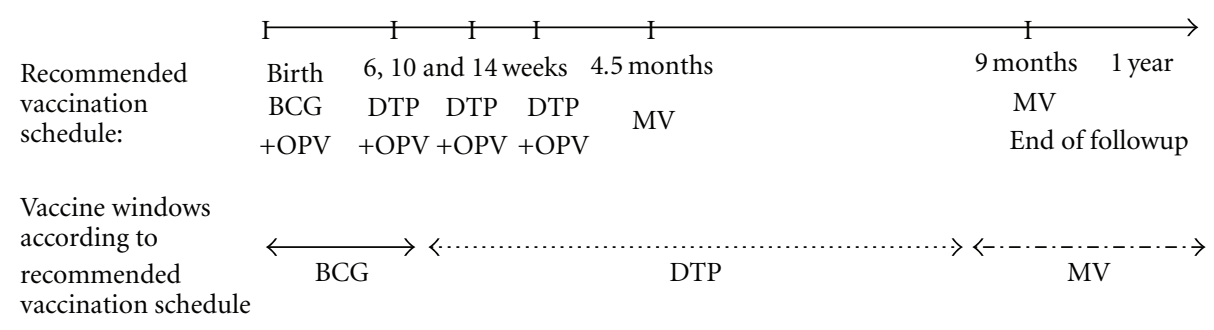

Vaccine windows according to vaccination schedule of the individual child:

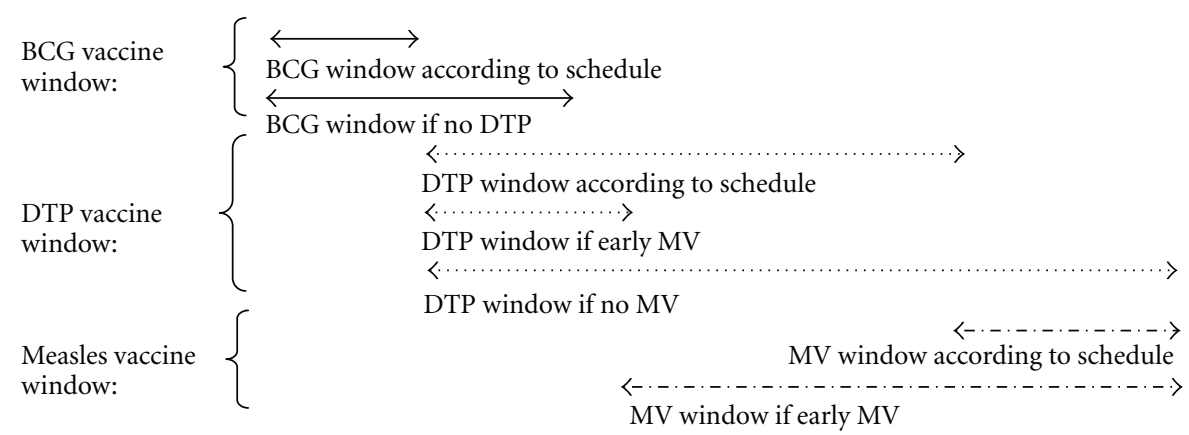

FIgURE 1: Vaccinations schedule and vaccine windows for infants in Guinea-Bissau.

therefore examined whether VAS administered with BCG at birth affected growth during the first year of life and furthermore whether neonatal VAS interacted with subsequent routine vaccines. All analyses were stratified by sex since neonatal VAS may have sex-differential effects [7, 9, 24].

\section{Subjects and Methods}

2.1. Study Population. Bandim Health Project runs a Health and Demographic Surveillance System (HDSS) in six suburban districts of the capital of Guinea-Bissau, Bissau. The present study was carried out within a neonatal VAS trial which has been described in detail elsewhere [7]. Briefly, mothers who gave birth to infants of at least $2500 \mathrm{~g}$ were invited to participate in the trial when the child was due to be BCG vaccinated at the maternity wards or health centres. A trained assistant explained the study. Provided maternal consent, children were randomised to vitamin A or placebo. VAS was administered orally as $0.5 \mathrm{~mL}$ of oil containing $50000 \mathrm{IU}$ vitamin $\mathrm{A}$ as retinyl palmitate and $10 \mathrm{IU}$ vitamin $\mathrm{E}$ and placebo was $0.5 \mathrm{~mL}$ of oil containing only $10 \mathrm{IU}$ vitamin E. The code was broken when all enrolled children had reached 12 months of age.

2.2. Anthropometrics. The effect on growth was studied in a subcohort comprising all children aged 0 to 6 weeks of age when enrolled between July 1, 2004 and November 28, 2004, when the main trial ended. Hence, all children had been enrolled in the rainy season from June to November. We aimed to include 800 children. Children were visited every 6 weeks. Anthropometric measurements were initiated October 20,2004. The oldest of these children were therefore measured for the first time at 3 months, corresponding to having missed one visit by the anthropometry team.
Measurements were made by two trained field assistants who visited the home of the child. The length of the child was measured supine using a wooden measuring board. The weight of the undressed child was measured to the nearest $20 \mathrm{~g}$ using an electronic scale (SECA Model 835). Children, who were temporarily absent when the house was visited, were visited later the same or the following day, whereas children travelling were only visited at the following round. Children who moved within the study area were localized using the HDSS and visited at the new address.

2.3. Vaccine Information. The recommended immunisation schedule in Guinea-Bissau for normal-birth-weight infants during the conduct of the study was BCG and oral polio vaccine (OPV) at birth, 3 doses of DTP and OPV at 6, 10, and 14 weeks, and MV at 9 months of age (Figure 1). The actual timing of the vaccines varied depending on when the mother took the child to the health centre for vaccination. Due to periods with lack of OPV, some children received only BCG at birth. This was registered on the inclusion form. The anthropometry team collected information on vaccination status of the child when conducting the measurements. Furthermore, vaccination status was assessed through the HDSS at the home visits every 3 months, and all vaccines administered at the health centres in the study area are registered daily. The vaccine dates collected by the anthropometry team have been validated against these routine data.

Early Measles Vaccine Trial. Some of the children in the present VAS at birth trial were later enrolled in an early measles vaccine trial. Within that trial children, who had received all three DTP vaccines, were randomised to early MV at 4.5 months of age or no early MV. All children received MV at 9 months of age as recommended [17]. 
TABLE 1: Baseline characteristics of all children included in the growth cohort by randomisation group.

\begin{tabular}{|c|c|c|}
\hline & $\begin{array}{c}\text { Vitamin A } \\
\text { Number (\%) }\end{array}$ & $\begin{array}{c}\text { Placebo } \\
\text { Number (\%) }\end{array}$ \\
\hline Number & 403 & 405 \\
\hline Sex (Male) & $198(49)$ & $194(48)$ \\
\hline No oral polio vaccine at enrolment & $88(22)$ & $83(20)$ \\
\hline \multicolumn{3}{|l|}{ Electricity in the household* } \\
\hline Yes & $142(35)$ & $140(35)$ \\
\hline No & $246(61)$ & $261(64)$ \\
\hline \multicolumn{3}{|l|}{ Bathroom* } \\
\hline Inside the house & $63(16)$ & $56(14)$ \\
\hline Outside the house & $324(80)$ & $343(85)$ \\
\hline None & $0(0)$ & $2(0)$ \\
\hline \multicolumn{3}{|l|}{ Maternal education* } \\
\hline Any & $256(64)$ & $239(59)$ \\
\hline None & $122(30)$ & $147(36)$ \\
\hline \multicolumn{3}{|l|}{ Birth order* } \\
\hline Primipara & $109(27)$ & $113(28)$ \\
\hline 2nd-3rd & $156(39)$ & $142(35)$ \\
\hline \multirow[t]{2}{*}{4 th or higher } & $124(31)$ & $147(36)$ \\
\hline & Mean (SD) & Mean (SD) \\
\hline Birth weight/kg & $3.19(0.44)$ & $3.23(0.40)$ \\
\hline Weight at incl. $/ \mathrm{kg}$ & $3.19(0.51)$ & $3.22(0.51)$ \\
\hline Weight for age at incl./z-score & $-0.47(0.95)$ & $-0.38(0.88)$ \\
\hline Length at inclusion & $50.2(2.3)$ & $50.1(2.3)$ \\
\hline Length for age at incl./z-score & $-0.08(1.00)$ & $-0.12(0.93)$ \\
\hline Maternal age/years & $25.0(5.7)$ & $25.1(5.6)$ \\
\hline Maternal arm circumference/mm & $255(33)$ & $254(30)$ \\
\hline
\end{tabular}

* Numbers do not add up due to a few with missing information.

2.4. Analytical Strategy and Statistics. We used two complementary approaches to assess growth patterns in the first year of life. All analyses were conducted by sex as neonatal VAS may have sex-differential effects [24, 25]. For the both approaches we compared the individual measurements to the 2006 WHO growth reference [26]. Z-scores, that is, the number of standard deviations that a measurement differs from the reference curve for length for age, weight for age and weight for length were derived and children were classified as stunted (length-for-age $z$-score $<-2$ ) and underweight (weight-for-age $\mathrm{z}$-score $<-2$ ) at all measured time points. Our first approach was to study whether neonatal VAS affected weight for age and length for age at 6 months (when the children would be eligible to receive VAS in campaigns; range 4-7 months) and at 12 months (when followup stopped; range 10-14 months). We compared the $\mathrm{z}$-scores by linear regression and calculated relative risks of being stunted or underweight.

In the second approach, we studied the effect of VAS in the time windows where the BCG, DTP, and MV were the last vaccines to investigate whether the effect of neonatal VAS with BCG changed when the immune system was influenced sequentially by different vaccines (Figure 1). We focused on changes in weight for length which better catch short-term changes in growth [27]. The vaccine windows began when the child received the vaccine in question and ended one week after vaccination with a different vaccine (or at a chosen cut-off age if no subsequent vaccine was given). Growth in the BCG window was defined as the difference in the weightfor-length z-score from inclusion to within one week after the first DTP vaccine (DTP1); if no DTP was given, the last measurement before 4 months of age was used. Similarly, growth in the DTP window was defined as the difference in weightfor-length z-score between the first measurement after DTP1 and the last measurement within one week after the first MV. If the child had not received an MV before 12 months of age the last obtained measurement before/at 12 months of age was used. Finally, the growth in the MV window was defined as the difference in the first z-score measured after MV and the last measurement before/at 12 months of age (Figure 1). We also conducted the analysis on growth in the MV window stratified by reception of early MV at 4.5 months and 9 months of age or routine MV at 9 months.

To contribute in one of the vaccines window analyses a child should thus have at least two measurements in the time window. The change in weight for length between the first and the last measurements in a vaccine window was compared by linear regression. A mixed-effect regression model for repeated measures was used to examine whether the effect of VAS differed between the vaccine windows.

Others have found an effect of VAS on growth in vitaminA-deficient children [28]. We did not measure vitaminAstatus at the time of supplementation. It has been shown consistently that vitamin A status varies with birth weight, that is, smaller newborns have poorer vitamin A status [2932]. In addition boys have lower cord blood retinol concentration [29]. We therefore investigated whether socioeconomic status and weight in the first week of life modified the effect of VAS on weight and length for age at 6 and 12 months and on change in weight for length in the vaccine windows.

\section{Results}

We enrolled 808 children. Of these 490 were later enrolled in the early measles vaccine trial. At baseline the vitamin $\mathrm{A}$ and placebo groups were comparable (Table 1). Children included in the growth cohort, but never measured $(n=83)$, showed the same distribution of background factors (data not shown).

We aimed to visit the children approximately every 6 weeks. Mean time between two visits was 46.3 days (standard deviation $(\mathrm{SD})=9.3$ ). The intervals between two visits did not differ by randomisation group $(P=0.46)$. An average of $68 \%$ of the children was found at home to be measured at these visits (VAS: 67\%, Placebo: 68\%). The majority of those not measured were travelling at the time of the visit. Of the 808 children included in the growth cohort, 725 (90\%) were measured at least once between inclusion and 12 months of age. The remaining 83 were not measured for reasons stated in Figure 2. Twenty-seven children died before 1 year of 


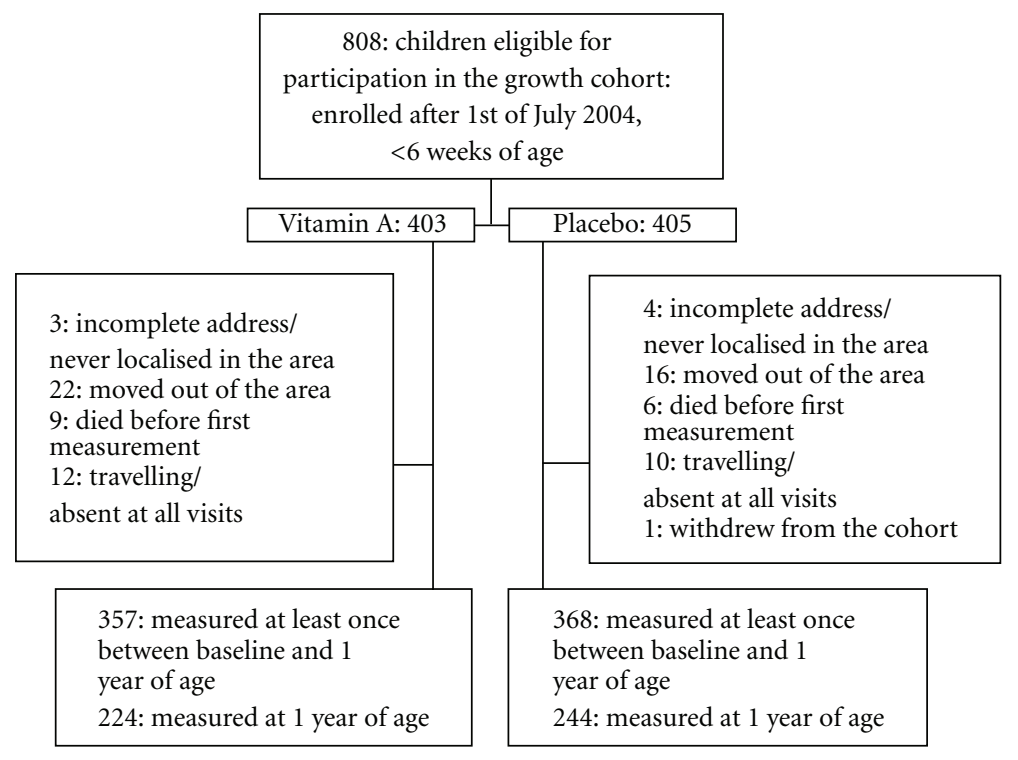

FIgURE 2: Flow of children through the study.

age (19 VAS: 10 boys, 9 girls; 8 placebo: 5 boys, 3 girls), corresponding to a relative risk (RR) of loss to followup due to death for VAS versus placebo of 2.38 (1.06-5.39).

3.1. First Approach: Anthropometric Status at 6 and 12 Months. At 6 months of age there was no overall effect of VAS at birth on length-for-age z-score $(P=0.58)$ or weightfor-age $z$-score $(P=0.79)$, and the effect of VAS did not differ between boys and girls, $P=0.28$ for length for age and $P=0.25$ for weight for age. VAS tended to increase the proportion of stunted girls, but to decrease the proportion of stunted boys $(P$ for interaction between VAS and sex $=$ 0.11 ) (Table 2). At 12 months there was no overall effect of VAS at birth on length for age $(P=0.28)$ or weight for age $(P=0.35)$ (Table 2$)$. The majority of the children had attained a length below the reference median (90\%), resulting in an average length for age of below -1 SD in both the VAS and the placebo groups. VAS tended to benefit the growth in boys during the first year of life but this was not found in girls $(P$ for interaction between sex and VAS $=0.27$ for being stunted and 0.06 for being underweight).

3.2. Second Approach: Growth by Vaccination Status. In the time window of the BCG vaccination there was an overall significant beneficial effect of VAS due to a significant beneficial effect for boys (Table 3 ). In the time window of the DTP vaccine there was no overall effect of VAS at birth, but a tendency for a negative effect was seen for girls $(P=0.12)$. In the time window of the MV there was no effect overall or in either sex. The overall effect of VAS in the BCG window was different from the effect in the DTP window $(P=0.03)$, but did not differ from the effect in the MV window $(P=0.11)$. Furthermore, the effect of neonatal VAS tended to differ between the DTP and MV windows for girls $(P=0.09)$, but not for boys $(P=0.67)$. The beneficial effect in the MV window may have been slightly stronger among the children who received an early MV and another one at 9 months of age. In none of the time windows the effect of neonatal VAS differed significantly between boys and girls $(P$ for interaction between VAS and sex $=0.28,0.18$, and 0.61 in the BCG, DTP and MV windows, resp.).

Due to shortage of vaccine $21 \%$ of the children did not receive OPV at enrolment. The effect of neonatal VAS on growth at 6 or 12 months of age or in the vaccine windows did not differ by reception of OPV at enrolment (data not shown).

3.3. Growth by Markers of Vitamin A Status. Stratification by the background factors presented in Table 1 did not indicate that the effect of VAS on growth parameters at 6 or 12 months or in the vaccine windows differed by socioeconomic groups (data not shown).

In tertiles of weight at enrolment for children enrolled in the first week of life, the effect of VAS was different in boys and girls. VAS tended to have a positive effect in the lightest girls in the BCG window immediately after supplementation, whereas the effect tended to be negative in the heavier girls $(P=0.10$ for interaction between VAS and enrolment weight in the BCG window). In contrast, VAS tended to have a negative effect in the lightest boys and a positive in the heavier boys $(P=0.10$ for interaction between enrolment weight and VAS in boys). This resulted in a statistically significant 3-way interaction between sex, VAS, and enrolment weight with respect to change in weight for length in the BCG window $(P=0.01)$. This effect was not sustained at 12 months of age (data not shown).

\section{Discussion}

Children in Guinea-Bissau do not fulfil their growth potential during the first year of life according to the WHO references. More than half were below minus 1 length-for-age 


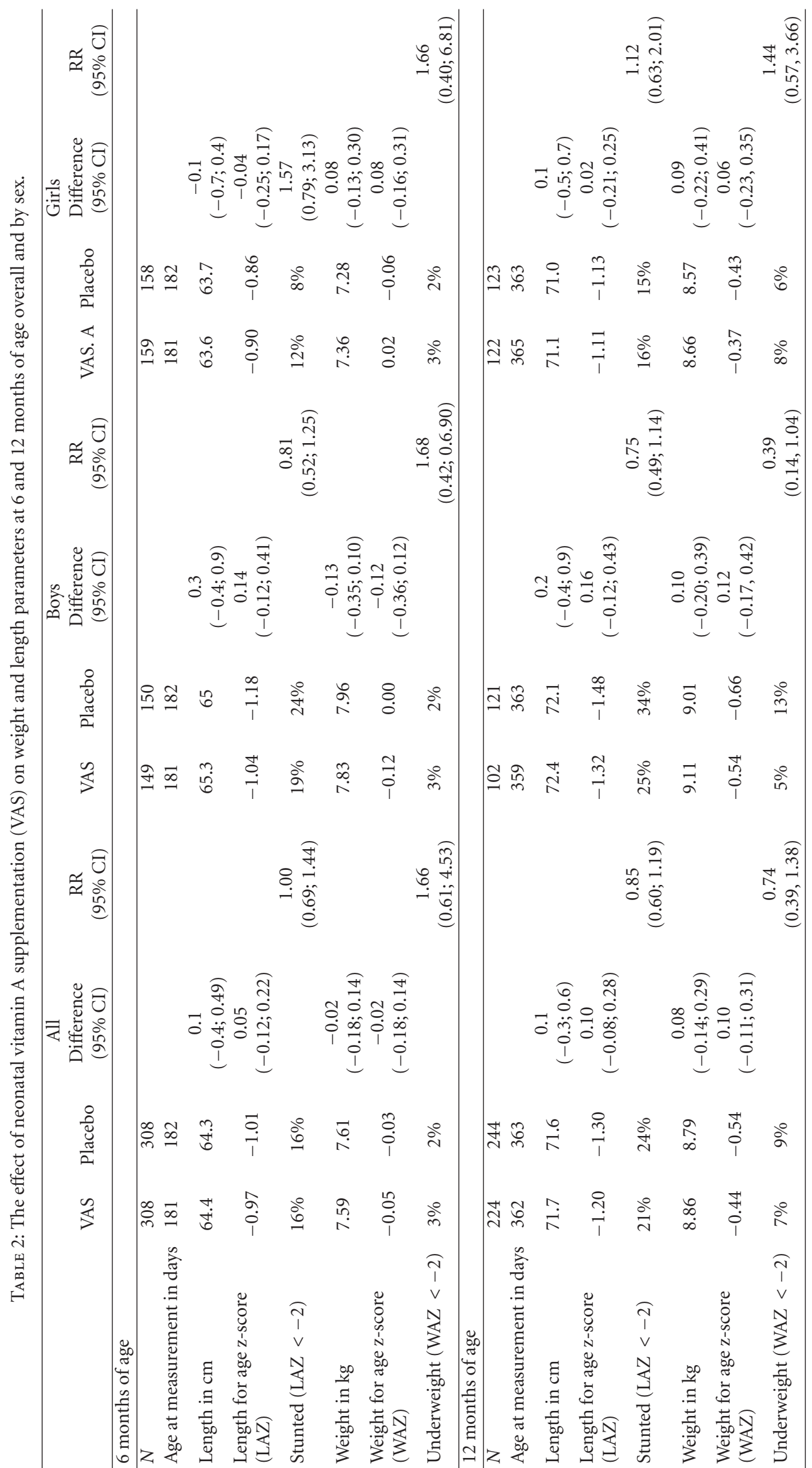




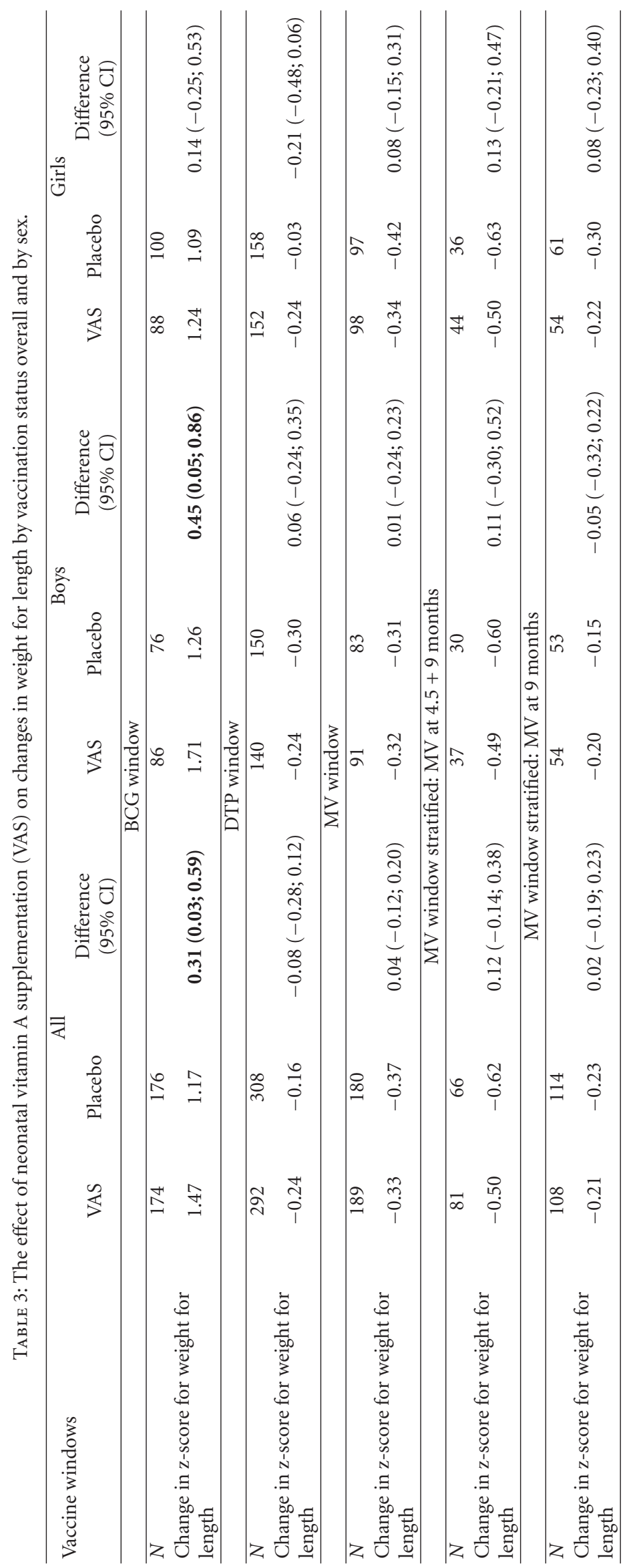


Z-score at 12 months of age. Overall there was no effect of neonatal VAS on growth at 6 and 12 months of age, but we observed a tendency towards a beneficial effect of VAS for boys but not for girls. The effect of VAS differed by vaccination status: VAS was beneficial as long as BCG was the most recent vaccine. The effect in the BGC window was significantly different from the effect in the DTP window where neonatal VAS tended to have a negative effect in girls. The effect of VAS tended also to differ between the DTP and MV windows for girls.

4.1. Strengths and Limitations. We measured children enrolled in the randomised trial at home visits, limiting the dropout rate and minimising the risk of selection bias associated with children having to report to a health centre. However, the frequency of travelling and movements in urban GuineaBissau is high, and only around two-thirds of the children were measured during each round. For 10\% we did not achieve a measurement at any time point during the first year of life. However, the baseline characteristics of these children did not differ from those of the measured children. In any growth study it could be argued that the results could be skewed if mortality during followup differed between the groups. In the present study mortality during followup was significantly higher in the vitamin A than in the placebo group, in particular among girls. This, if anything, would tend to mask a negative effect of VAS on growth in girls.

The study of mortality revealed a strong interaction between season of supplementation and VAS, with VAS being detrimental for girls in the rainy season, and most beneficial for boys in the dry season [7]. Unfortunately, as this was an unexpected finding, it was not taken into account when planning the present study and all enrolments were in the rainy season Hence, we could study possible effect modification by season of enrolment.

4.2. Interpretation. We set out to examine the effect of VAS with BCG on the growth patterns in the first year of life including shifts in effect when the immune system is influenced sequentially by BCG, DTP, and then MV. As expected we found a positive effect in the BCG window. It seemed most pronounced for boys. In the time window of DTP, girls had a tendency for a negative effect of VAS, whereas boys still may have a slight benefit of being assigned to the VAS group. This may be due to a nonbeneficial interaction of vitamin A with DTP in girls, consistent with the mortality results [23]. The effect of VAS differed significantly between the BCG and the DTP windows, and there was a borderline significant difference between the DTP and MV windows in girls.

It could be argued that that positive effect of VAS in the BCG window was due to prevention/treatment of VAD, and this effect waned as vitamin A was consumed. However, this would not account for the tendency for a negative effect in the DTP window in girls. Others have found an effect of VAS on growth limited to groups with low vitamin A status [33], but this pattern is not supported by our data. In the present study boys had a more beneficial effect than girls in the BCG window; however, it was the boys with higher inclusion weights who seemed to benefit more from VAS in the BCG window. The opposite was the case for girls. If the effect is different in boys and girls, with a tendency for most beneficial effect in the least deficient boys and the most deficient girls immediately after supplementation, it seems unlikely that the beneficial effect of VAS at birth in certain subgroups was merely due to a reduction in VAD. Furthermore, if the effect of VAS on growth depended on the prevalence of VAD, we would expect a more pronounced difference between vitamin A and placebo among those of lower socioeconomic status [34-36]. We found no indication that a beneficial effect was seen in subgroups with worse socioeconomic status. Hence the effect of VAS on growth may be due to an interaction with the immune system, VAS affecting susceptibility to infections differently when BCG and DTP, respectively, have been administered as the most recent vaccine.

\section{Conclusion}

The effect on growth of $50000 \mathrm{IU}$ vitamin A given at birth with BCG vaccine appears to be beneficial for boys, whereas it tended to be negative for girls who had received DTP. The mechanisms behind these effects are unknown, but are unlikely to be explained by prevention of vitamin A deficiency.

\section{Author's Contribution}

Christine Stabell Benn, Amabelia Rodrigues, and Peter Aaby designed the study of neonatal VAS. Christine Stabell Benn and Peter Aaby initiated the study of neonatal VAS. Ane Bærent Fisker and Birgitte Rode Diness participated in supervising recruitment and initiated and supervised the followup for growth. Cesario Martins was responsible for the trial of early MV and contributed the data on early measles vaccinations. Ane Bærent Fisker and Bo Martin Bibby were responsible for the statistical analysis. Ane Bærent Fisker wrote the first draft of the paper. All authors contributed to and approved the final version of the paper.

\section{Conflict of Interests}

The authors declare no conflict of interests.

\section{Disclosure}

The funding agencies had no role in the study design, data collection, data analysis, data interpretation, or the writing of the paper.

\section{Acknowledgments}

This work was supported by the EU (ICA4-CT-2002-10053), the Danish Medical Research Council, University of Copenhagen, March of Dimes, and the Ville Heise Foundation. Ane Bærent Fisker received funding from the Danish Research Council, Codan foundation and "Grosserer Ludvig Berlin og Frøken Marie Poulsens Fond." The Bandim Health Project 
received support from DANIDA and the Danish National Research Foundation. Peter Aaby holds a research professorship grant from the Novo Nordisk Foundation.

\section{References}

[1] W. W. Fawzi, M. G. Herrera, W. C. Willett, P. Nestel, A. El Amin, and K. A. Mohamed, "Dietary vitamin A intake in relation to child growth," Epidemiology, vol. 8, no. 4, pp. 402-407, 1997.

[2] G. J. Fuchs, S. Ausayakhun, S. Ruckphaopunt, A. Tansuhaj, and R. M. Suskind, "Relationship between vitamin A deficiency, malnutrition, and conjunctival impression cytology," American Journal of Clinical Nutrition, vol. 60, no. 2, pp. 293-298, 1994.

[3] U. Ramakrishnan, N. Aburto, G. McCabe, and R. Martorell, "Multimicronutrient interventions but not vitamin A or iron interventions alone improve child growth: results of 3 metaanalyses," Journal of Nutrition, vol. 134, no. 10, pp. 2592-2602, 2004.

[4] M. K. Schmidt, S. Muslimatun, W. Schultink, C. E. West, and J. G. A. J. Hautvast, "Randomised double-blind trial of the effect of vitamin A supplementation of Indonesian pregnant women on morbidity and growth of their infants during the first year of life," European Journal of Clinical Nutrition, vol. 56, no. 4, pp. 338-346, 2002.

[5] M. K. Schmidt, S. Muslimatun, C. E. West, W. Schultink, and J. G. A. J. Hautvast, "Vitamin A and iron supplementation of Indonesian pregnant women benefits vitamin A status of their infants," British Journal of Nutrition, vol. 86, no. 5, pp. 607615,2001 .

[6] J. H. Humphrey, T. Agoestina, A. Juliana et al., "Neonatal vitamin A supplementation: effect on development and growth at 3 y of age," American Journal of Clinical Nutrition, vol. 68, no. 1, pp. 109-117, 1998.

[7] C. S. Benn, B. R. Diness, A. Roth et al., "Effect of $50000 \mathrm{IU}$ vitamin A given with BCG vaccine on mortality in infants in Guinea-Bissau: randomised placebo controlled trial," British Medical Journal, vol. 336, no. 7658, pp. 1416-1420, 2008.

[8] L. C. Malaba, P. J. Iliff, K. J. Nathoo et al., "Effect of postpartum maternal or neonatal vitamin A supplementation on infant mortality among infants born to HIV-negative mothers in Zimbabwe," American Journal of Clinical Nutrition, vol. 81, no. 2, pp. 454-460, 2005.

[9] J. H. Humphrey, T. Agoestina, L. Wu et al., "Impact of neonatal vitamin A supplementation on infant morbidity and mortality," Journal of Pediatrics, vol. 128, no. 4, pp. 489-496, 1996.

[10] L. Rahmathullah, J. M. Tielsch, R. D. Thulasiraj et al., "Impact of supplementing newborn infants with vitamin A on early infant mortality: community based randomised trial in southern India," British Medical Journal, vol. 327, no. 7409, pp. 254 260, 2003.

[11] R. D. W. Klemm, A. B. Labrique, P. Christian et al., "Newborn vitamin a supplementation reduced infant mortality in rural bangladesh," Pediatrics, vol. 122, no. 1, pp. e242-e250, 2008.

[12] C. S. Benn, C. Balé, H. Sommerfelt, H. Friis, and P. Aaby, "Hypothesis: vitamin A supplementation and childhood mortality: amplification of the non-specific effects of vaccines?" International Journal of Epidemiology, vol. 32, no. 5, pp. 822828, 2003.
[13] C. S. Benn, C. Martins, A. Rodrigues et al., "The effect of vitamin A supplementation administered with missing vaccines during national immunization days in Guinea-Bissau," International Journal of Epidemiology, vol. 38, no. 1, pp. 304311, 2009.

[14] P. Aaby, B. Samb, F. Simondon, A. M. C. Seck, K. Knudsen, and H. Whittle, "Non-specific beneficial effect of measles immunisation: analysis of mortality studies from developing countries," British Medical Journal, vol. 311, no. 7003, pp. 481485, 1995.

[15] I. Kristensen, P. Aaby, and H. Jensen, "Routine vaccinations and child survival: follow up study in Guinea-Bissau, West Africa," British Medical Journal, vol. 321, no. 7274, pp. 1435$1439,2000$.

[16] P. Aaby, A. Bhuiya, L. Nahar, K. Knudsen, A. de Francisco, and M. Strong, "The survival benefit of measles immunization may not be explained entirely by the prevention of measles disease: a community study from rural Bangladesh," International Journal of Epidemiology, vol. 32, no. 1, pp. 106-115, 2003.

[17] P. Aaby, C. L. Martins, M. L. Garly et al., "Non-specific effects of standard measles vaccine at 4.5 and 9 months of age on childhood mortality: randomised controlled trial," British Medical Journal, vol. 341, article c6495, 2010.

[18] P. Aaby, A. Roth, H. Ravn et al., " Randomized trial of BCG vaccination at birth to low-birth-weight children: beneficial nonspecific effects in the neonatal period?" Journal of Infectious Diseases, vol. 204, pp. 245-252, 2011.

[19] A. E. Roth, C. Stabell Benn, H. Ravn et al., "Effect of revaccination with BCG in early childhood on mortality: randomised trial in Guinea-Bissau," British Medical Journal, vol. 340, article c671, 2010.

[20] A. Roth, H. Jensen, M. L. Garly et al., "Low birth weight infants and Calmette-Guérin bacillus vaccination at birth: community study from Guinea-Bissau," Pediatric Infectious Disease Journal, vol. 23, no. 6, pp. 544-550, 2004.

[21] P. Aaby, H. Jensen, A. Rodrigues et al., "Divergent female-male mortality ratios associated with different routine vaccinations among female-male twin pairs," International Journal of Epidemiology, vol. 33, no. 2, pp. 367-373, 2004.

[22] P. Aaby, H. Vessari, J. Nielsen et al., "Sex differential effects of routine immunizations and childhood survival in rural Malawi," Pediatric Infectious Disease Journal, vol. 25, no. 8, pp. 721727, 2006.

[23] C. S. Benn, A. Rodrigues, M. Yazdanbakhsh et al., "The effect of high-dose vitamin A supplementation administered with BCG vaccine at birth may be modified by subsequent DTP vaccination," Vaccine, vol. 27, no. 21, pp. 2891-2898, 2009.

[24] C. S. Benn, A. B. Fisker, B. R. Diness, and P. Aaby, "Neonatal vitamin A supplementation: sex-differential effects on mortality?" Journal of Infectious Diseases, vol. 194, no. 5, pp. 719-721, 2006.

[25] C. S. Benn, C. Martins, A. Rodrigues, H. Jensen, I. M. Lisse, and P. Aaby, "Randomised study of effect of different doses of vitamin A on childhood morbidity and mortality," British Medical Journal, vol. 331, no. 7530, pp. 1428-1432, 2005.

[26] World Health Organization MRSG, "WHO child growth standards: length/height-for-age, weight-for-age, weight-forlength, weight-for-height and body mass index-for-age," Methods and Development. Available at: http://www.who.int/ childgrowth/standards/Technical_report.pdf.

[27] A. Briend, K. Z. Hasan, K. M. A. Aziz, B. A. Hoque, and F. J. Henry, "Measuring change in nutritional status: a comparison of different anthropometric indices and the sample sizes required," European Journal of Clinical Nutrition, vol. 43, no. 11, pp. 769-778, 1989. 
[28] H. Hadi, R. J. Stoltzfus, M. J. Dibley et al., "Vitamin A supplementation selectively improves the linear growth of Indonesian preschool children: results from a randomized controlled trial," American Journal of Clinical Nutrition, vol. 71, no. 2, pp. 507-513, 2000.

[29] A. M. Tolba, F. M. Hewedy, A. M. Al-Senaidy, and A. A. Al-Othman, "Neonates' vitamin a status in relation to birth weight, gestational age, and sex," Journal of Tropical Pediatrics, vol. 44, no. 3, pp. 174-177, 1998.

[30] D. A. Adelekan, C. A. Northrop-Clewes, J. A. Owa, A. O. Oyedeji, A. A. Owoeye, and D. I. Thurnham, "Use of biomarkers of sub-clinical infection, nutrition and neonatal maturity to interpret plasma retinol in Nigerian neonates," British Journal of Nutrition, vol. 90, no. 2, pp. 353-361, 2003.

[31] K. Ghebremeskel, L. Burns, T. J. Burden et al., "Vitamin A and related essential nutrients in cord blood: relationships with anthropometric measurements at birth," Early Human Development, vol. 39, no. 3, pp. 177-188, 1994.

[32] P. H. C. Rondo, R. Abbott, L. C. Rodrigues, and A. M. Tomkins, "Vitamin A, folate, and iron concentrations in cord and maternal blood of intra-uterine growth retarded and appropriate birth weight babies," European Journal of Clinical Nutrition, vol. 49, no. 6, pp. 391-399, 1995.

[33] H. Hadi, R. J. Stoltzfus, M. J. Dibley et al., "Vitamin A supplementation selectively improves the linear growth of Indonesian preschool children: results from a randomized controlled trial," American Journal of Clinical Nutrition, vol. 71, no. 2, pp. 507-513, 2000.

[34] J. O. Alvarez, E. Salazar-Lindo, J. Kohatsu, P. Miranda, and C. B. Stephensen, "Urinary excretion of retinol in children with acute diarrhea," American Journal of Clinical Nutrition, vol. 61, no. 6, pp. 1273-1276, 1995.

[35] D. Bishai, K. C. S. Kumar, H. Waters et al., "The impact of vitamin A supplementation on mortality inequalities among children in Nepal," Health Policy and Planning, vol. 20, no. 1, pp. 60-66, 2005.

[36] J. Nielsen, C. S. Benn, C. Balé, C. Martins, and P. Aaby, "Vitamin A supplementation during war-emergency in GuineaBissau 1998-1999," Acta Tropica, vol. 93, no. 3, pp. 275-282, 2005. 


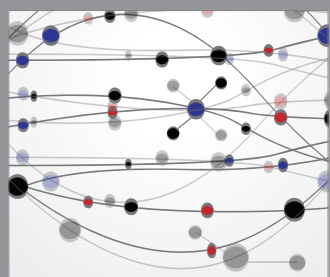

The Scientific World Journal
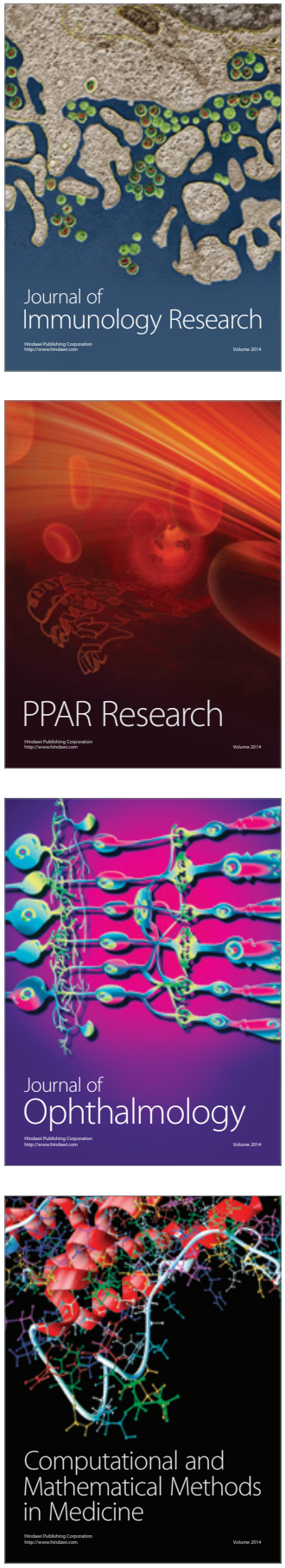

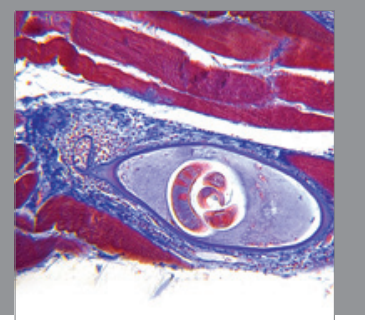

Gastroenterology

Research and Practice
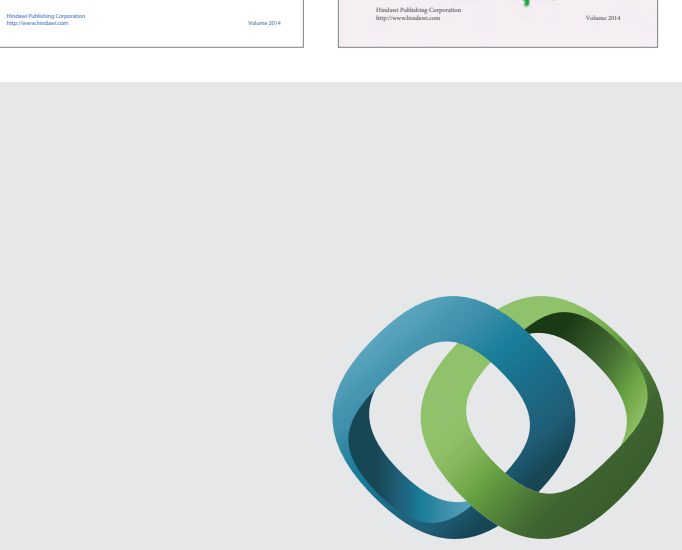

\section{Hindawi}

Submit your manuscripts at

http://www.hindawi.com
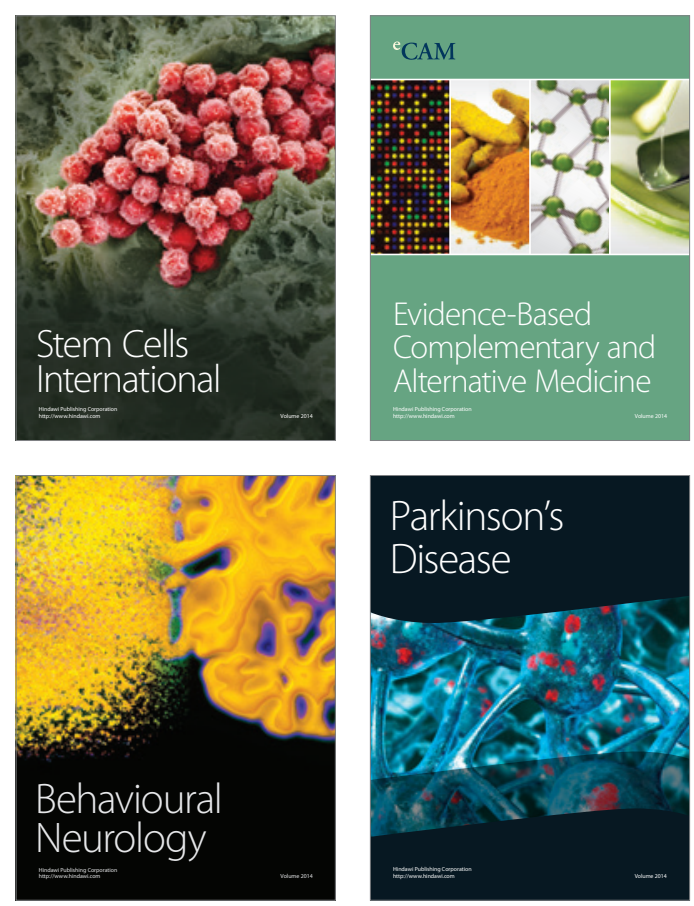

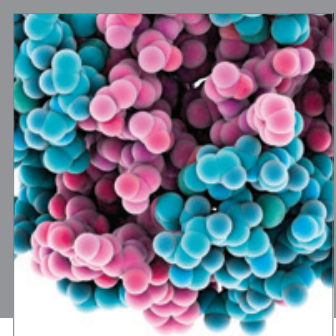

Journal of
Diabetes Research

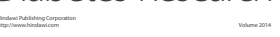

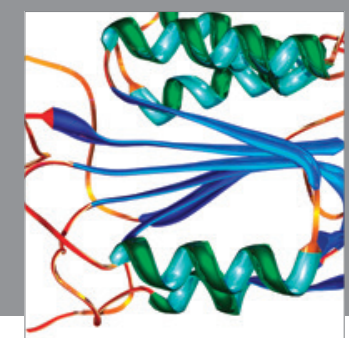

Disease Markers
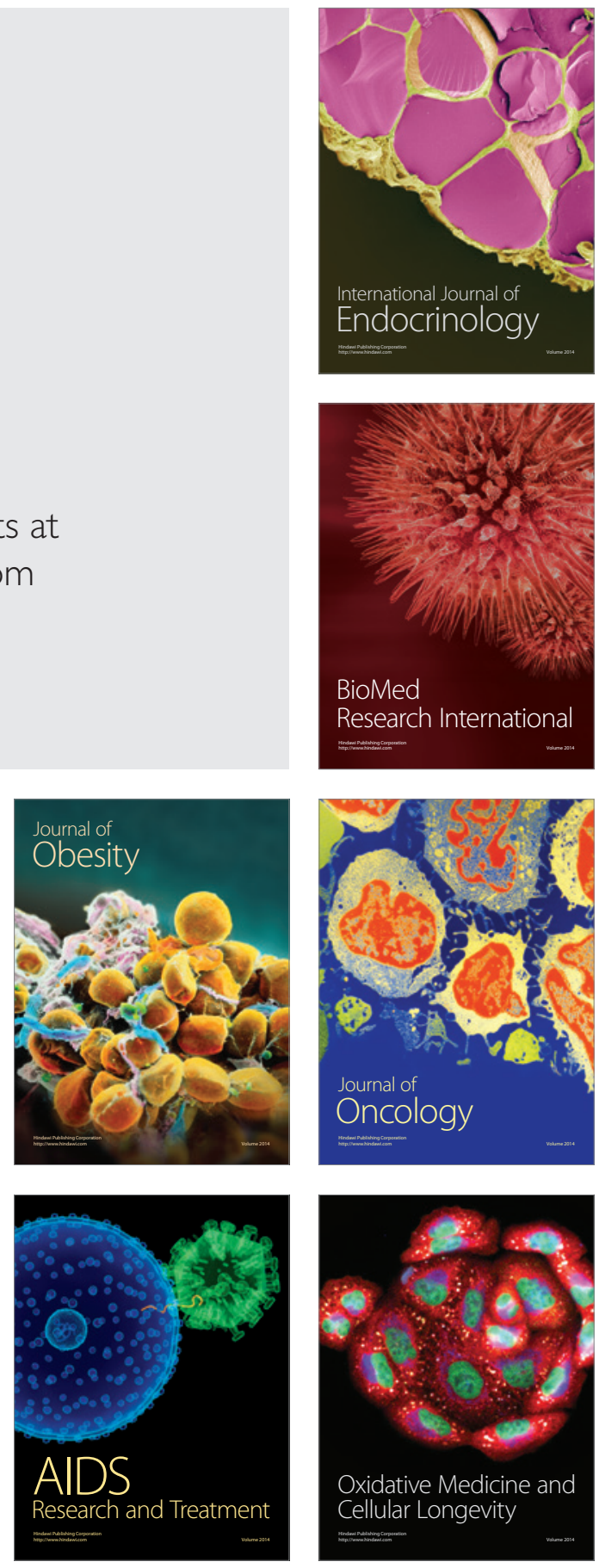